\title{
Usefulness of Transcranial Magnetic Stimulation to Assess Motor Function in Patients With Parkinsonism
}

\author{
Jaechan Park, $\mathrm{MD}^{1}$, Won Hyuk Chang, $\mathrm{MD}, \mathrm{PhD}^{1}$, Jin Whan Cho, $\mathrm{MD}, \mathrm{PhD}^{2}$, Jinyoung Youn, $\mathrm{MD}, \mathrm{PhD}^{2}$, \\ Yun Kwan Kim ${ }^{1}$, Sun Woong Kim, MD ${ }^{1}$, Yun-Hee Kim, MD, $\mathrm{PhD}^{1,3,4}$
}

\begin{abstract}
${ }^{1}$ Department of Physical and Rehabilitation Medicine, Center for Prevention and Rehabilitation, Heart Vascular and Stroke Institute, Samsung Medical Center, Sungkyunkwan University School of Medicine, Seoul; ${ }^{2}$ Department of Neurology, Samsung Medical Center, Sungkyunkwan University School of Medicine, Seoul; Departments of ${ }^{3} \mathrm{Health}$ Science and Technology and

${ }^{4}$ Medical Device Management \& Research, SAIHST, Sungkyunkwan University, Seoul, Korea
\end{abstract}

Objective To investigate the clinical significance of upper and lower extremity transcranial magnetic stimulation (TMS)-induced motor evoked potentials (MEPs) in patients with parkinsonism.

Methods Twenty patients ( 14 men, 6 women; mean age $70.5 \pm 9.1$ years) suffering from parkinsonism were included in this study. All participants underwent single-pulse TMS session to assess the corticospinal excitability of the upper and lower extremity motor cortex. The resting motor threshold (RMT) was defined as the lowest stimulus intensity able to evoke MEPs of an at least $50 \mu \mathrm{V}$ peak-to-peak amplitude in 5 of 10 consecutive trials. Five sweeps of MEPs at $120 \%$ of the RMT were performed, and the mean amplitude and latency of the MEPs were calculated. Patients were also assessed using the Unified Parkinson's Disease Rating Scale part III (UPDRS-III) and the 5-meter Timed Up and Go (5m-TUG) test.

Results There was a significant positive correlation between the RMTs of MEPs in the upper and lower extremities $(\mathrm{r}=0.612, \mathrm{p}=0.004)$ and between the amplitude of MEPs in the upper and lower extremities $(\mathrm{r}=0.579, \mathrm{p}=0.007)$. The RMT of upper extremity MEPs showed a significant negative relationship with the UPDRS-III score (r=-0.516, $\mathrm{p}=0.020$ ). In addition, RMTs of lower extremity MEPs exhibited a negative relationship with the UPDRS-III score, but the association was not statistically significant $(r=-406, p=0.075)$.

Conclusion These results indicated that the RMT of MEPs reflect the severity of motor dysfunction in patients with parkinsonism. MEP is a potential quantitative, electrodiagnostic method to assess motor function in patients with parkinsonism.

Keywords Transcranial magnetic stimulation, Motor evoked potentials, Parkinsonian disorders, Motor disorders

Received June 2, 2015; Accepted August 7, 2015

Corresponding author: Won Hyuk Chang

Department of Physical and Rehabilitation Medicine, Center for Prevention and Rehabilitation, Heart Vascular and Stroke Institute, Samsung Medical Center, Sungkyunkwan University School of Medicine, 81 Irwon-ro, Gangnam-gu, Seoul 06351, Korea

Tel: +82-2-3410-6068, Fax: +82-2-3410-0052, E-mail: wh.chang@samsung.com

() This is an open-access article distributed under the terms of the Creative Commons Attribution Non-Commercial License (http://creativecommons.org/ licenses/by-nc/4.0) which permits unrestricted noncommercial use, distribution, and reproduction in any medium, provided the original work is properly cited. Copyright (c) 2016 by Korean Academy of Rehabilitation Medicine 


\section{INTRODUCTION}

Transcranial magnetic stimulation (TMS)-induced motor evoked potentials (MEPs) have been widely used in various fields of neuroscience [1-3]. TMS-induced MEPs allow for the detection of corticospinal pathway disorders such as stroke, motor neuron diseases, multiple sclerosis, and spinal cord diseases $[4,5]$. Normative parameters of TMS-induced MEPs can be used to define a cutoff value that separates normal and abnormal measurements [6]. In addition, some parameters of TMS-induced MEPs are reported to correlate with motor cortical excitability $[6,7]$. Therefore, TMS-induced MEPs have been used to quantitatively assess the human motor system.

Parkinsonism is a clinical syndrome characterized by tremor, bradykinesia, rigidity, and postural instability due to various causes $[8,9]$. The most common diseases that manifest with parkinsonism are idiopathic Parkinson's disease (IPD) and other neurodegenerative diseases such as multiple system atrophy, progressive supranuclear palsy, and corticobasal degeneration [8,9]. Several functional assessment tools have been used to assess motor function in patients with parkinsonism, such as the Unified Parkinson's Disease Rating Scale part III (UPDRS-III), timed finger tapping task, and the Timed Up and Go test [10-12]. However, all functional assessment tools are limited in that they require patients to initiate effortful tasks.

TMS-induced MEPs have the potential to assess motor function in patients with parkinsonism; however, there is a lack of studies on the quantitative measurement potential of TMS-induced MEPs in patients with parkinsonism. The objective of this study was to investigate the clinical significance of upper and lower extremity TMS-induced MEPs in patients with parkinsonism.

\section{MATERIALS AND METHODS}

\section{Participants}

Twenty patients suffering from parkinsonism participated in this study. Diagnoses were based on medical history, physical examination, and neuroimaging studies conducted by experienced neurologists who specialize in movement disorders. We excluded patients with major psychiatric disease and those with pre-existing and active major neurological diseases other than parkinsonism.

This study was approved by the Institutional Review
Board of Samsung Medical Center, and written informed consent was obtained from all participants.

\section{Cortical excitability measurement}

During the TMS-induced MEPs, patients were seated comfortably in an armchair with their eyes open. Singlepulse TMS was applied over the dominant upper and lower primary motor cortex (M1) with a Magstim Rapid2 stimulator (Magstim, Carmarthenshire, UK). A Medelec Synergy electromyography/evoked potentials system (Oxford Instruments, Oxfordshire, UK) was used for monitoring and recording the activity of the dominant first dorsal interosseous muscle and tibialis anterior muscle in order to assess the upper and lower extremity motor cortical excitability, respectively. For the upper extremity M1, a 70-mm figure-of-eight coil was held tangentially to the scalp, with the handle pointing backward and laterally at $45^{\circ}$ from the mid-sagittal line. For the lower extremity M1, a double-cone coil was held tangentially to the scalp, with the handle pointing laterally at $90^{\circ}$ from the mid-sagittal line. Using TMS, the optimum position (hot spot) was defined as the site where the TMS-induced MEPs produced a maximum peak-to-peak amplitude in the dominant first dorsal interosseous and tibialis anterior muscle. The resting motor threshold (RMT) was defined as the lowest stimulus intensity able to evoke MEPs with an at least $50 \mu \mathrm{V}$ peak-to-peak amplitude in 5 of 10 consecutive trials $[13,14]$. Five sweeps of MEPs at $120 \%$ of the RMT were performed, and the mean amplitude and latency of the MEPs were calculated [13,14].

\section{Functional assessments}

Participants were evaluated using the UPDRS-III, which includes 14 items to assess the motor function of patients with parkinsonism [12,15]. In addition, the 5-meter Timed Up and Go (5m-TUG) test was conducted to measure ambulatory function. In patients with parkinsonism, a temporary and/or involuntary stoppage of movement is observed in the execution phase of walking and in turning movements [16]. Participants were asked to rise from a chair, walk forward for $5 \mathrm{~m}$, turn around, and walk back and sit in the same chair as fast as possible. This trial was video recorded, and the average time of 2 trials was calculated. Each functional assessment was performed by a single examiner who was blinded to the cortical excitability assessments. 


\section{Statistical analysis}

SPSS ver. 21.0 (IBM SPSS, Armonk, NY, USA) was used to perform statistical analyses. The Shapiro-Wilk test was used to determine the distributions of all continuous variables. The parameters of the TMS-induced MEPs did not showed a normal distribution $(\mathrm{p}<0.05$ by the ShapiroWilk test). Therefore, the Spearman correlation analysis was used to determine the relationships between corticospinal excitability and functional assessments. Differences were considered significant when $\mathrm{p}$-values were $<0.05$.

\section{RESULTS}

\section{Clinical characteristics}

The clinical characteristics of patients were shown in Table. 1 . The study group was composed of 14 men and 6 women, with a mean age of $70.5 \pm 9.1$ years who were suffering from parkinsonism.

Six and 14 patients were diagnosed with IPD and atypical parkinsonism, respectively. Their mean age was 70.5 years, and the mean duration of disease was 4.9 years. All participants exhibited a modified Hoehn and Yahr scale $[17]>2$.

\section{Relationship between upper and lower extremity cor-} tical excitability measurements

Fig. 1 showed the results of TMS-induced MEPs in the upper and lower extremities. There was a significant positive correlation between the RMTs of MEPs in the upper and lower extremities $(r=0.612, p=0.004)$ and between the amplitudes of MEPs in the upper and lower extremities ( $r=0.579, p=0.007$ ) (Fig. 1). However, there was no significant correlation between latency of the upper and lower extremity MEPs.

\section{Relationships between cortical excitability measure-} ment and functional assessments

For the correlation analysis between MEP parameters and functional assessments, the RMT of upper extremity MEPs showed a significant negative relationship with the UPDRS-III score $(\mathrm{r}=-0.516, \mathrm{p}=0.020)$. In addition, the RMT of lower extremity MEPs showed a negative relationship with the UPDRS-III score, but this result was not statistically significant $(r=-406, p=0.075)$. There were no significant correlations between the other MEP param-
Table 1. Clinical characteristics, cortical excitability measurements, and functional assessments of patients with parkinsonism

\begin{tabular}{|c|c|}
\hline Parameter & Value \\
\hline Age (yr) & $70.5 \pm 9.1(46-81)$ \\
\hline \multicolumn{2}{|l|}{ Sex (male:female) } \\
\hline Male & 14 \\
\hline Female & 6 \\
\hline \multicolumn{2}{|l|}{ Diagnosis } \\
\hline IPD & 6 \\
\hline Atypical parkinsonism & 14 \\
\hline Disease duration (yr) & $4.9 \pm 2.6(1-11)$ \\
\hline \multicolumn{2}{|l|}{$\begin{array}{l}\text { Modified Hoehn } \\
\text { and Yahr scale }\end{array}$} \\
\hline 2 & 1 \\
\hline 2.5 & 0 \\
\hline 3 & 11 \\
\hline 4 & 8 \\
\hline \multicolumn{2}{|l|}{ Motor evoked potentials } \\
\hline \multicolumn{2}{|l|}{ Upper extremity } \\
\hline $\begin{array}{l}\text { Resting motor } \\
\text { threshold (\%) }\end{array}$ & $36.8 \pm 9.5(24-65)$ \\
\hline Amplitude $(\mu \mathrm{V})$ & $587.77 \pm 376.35(166.9-1,844.2)$ \\
\hline Latency (ms) & $23.65 \pm 3.02(20.6-34.1)$ \\
\hline \multicolumn{2}{|l|}{ Lower extremity } \\
\hline $\begin{array}{l}\text { Resting motor } \\
\text { threshold (\%) }\end{array}$ & $43.6 \pm 10.1(30-60)$ \\
\hline Amplitude $(\mu \mathrm{V})$ & $426.74 \pm 212.02(112.3-945.5)$ \\
\hline Latency (ms) & $31.71 \pm 2.45(27.6-36.0)$ \\
\hline \multicolumn{2}{|l|}{ Functional assessments } \\
\hline UPDRS-III (score) & $21.8 \pm 10.9(7-43)$ \\
\hline 5m-TUG (s) & $59.80 \pm 63.69(13.7-270.1)$ \\
\hline
\end{tabular}

Values are presented as mean \pm standard deviation (range) or number.

IPD, idiopathic Parkinson's disease; UPDRS-III, Unified Parkinson's Disease Rating Scale part III; 5m-TUG, 5-meter Timed Up and Go test.

eters and functional assessments (Table 2).

\section{DISCUSSION}

We investigated the potential of TMS-induced MEPs to assess functional levels in patients with parkinsonism. The results of this study demonstrated that the RMT of upper MEPs have a significant relationship with mo- 

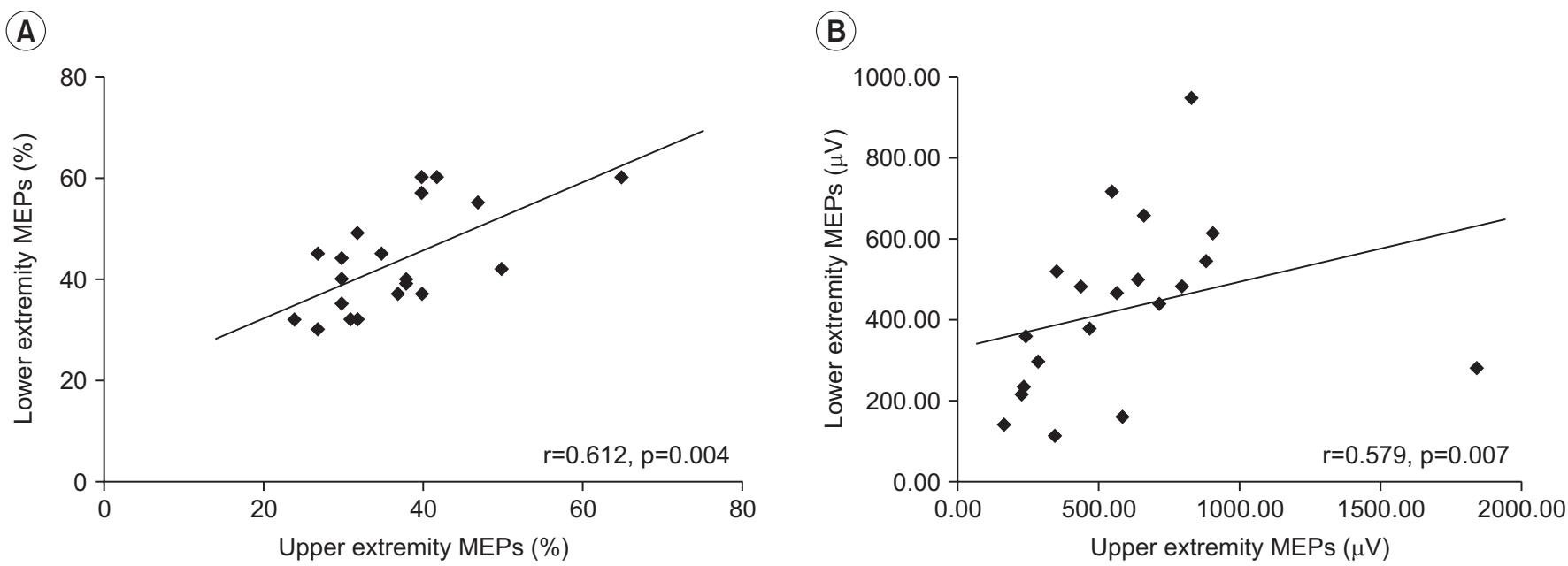

Fig. 1. Correlations between motor evoked potentials (MEPs) in upper and lower extremities. (A) Resting motor threshold and (B) amplitude.

Table 2. Correlations between motor evoked potentials and functional assessments

\begin{tabular}{lrr}
\hline & UPDRS-III & \multicolumn{1}{c}{ 5m-TUG } \\
\hline Upper extremity & & \\
Resting motor threshold & $-0.516(0.020)^{*}$ & $-0.145(0.542)$ \\
Amplitude & $0.111(0.642)$ & $-0.214(0.366)$ \\
Latency & $0.194(0.413)$ & $0.319(0.171)$ \\
Lower extremity & \\
\hline Resting motor threshold & $-0.406(0.075)$ & $0.257(0.273)$ \\
Amplitude & $0.108(0.649)$ & $-0.355(0.125)$ \\
Latency & $-0.297(0.204)$ & $-0.068(0.774)$ \\
\hline
\end{tabular}

Values are correlation coefficients ( $\mathrm{p}$-values).

UPDRS-III, Unified Parkinson's Disease Rating Scale part III; 5m-TUG, 5-meter Timed Up and Go test. ${ }^{*} \mathrm{p}<0.05$.

tor function in patients with parkinsonism. Thus, TMSinduced MEPs could be a used as a quantitative tool to assess motor function in patients with parkinsonism.

TMS-induced MEPs are a noninvasive and simple method for evaluating motor cortical excitability [6]. Recently, TMS-induced MEPs have become more popular to assess many CNS diseases. TMS-induced MEPs can be used as a tool to predict functional motor outcomes of stroke patients [18] and to monitor drug responses in neurological and psychiatric patients [19]. Atypical parkinsonism shares a similar set of symptoms with IPD, although the underlying causes of parkinsonism are numerous $[8,9]$. The primary pathophysiology of IPD is related to reduced activity of dopamine-secreting cells caused by cell death in the pars compacta region of the substantia nigra [9]. Due to increased activity of compensatory mechanisms, motor symptoms in IPD emerge only after a prolonged period of dopaminergic loss [20]. These compensations likely involve cerebral circuits outside of the basal ganglia including thalamocortical connections, the cerebellum, and sensorimotor cortex $[21,22]$. Therefore, TMS-induced MEPs allow analysis of changes in the motor cortex due to basal ganglia diseases such as IPD [22].

There are several parameters associated with TMS-induced MEPs, such as RMT, amplitude, and latency of MEPs [7]. RMT is defined as the lowest stimulus intensity of a single-pulse TMS that elicits a predefined, small motor response in the contralateral targeted muscle $[3,19,23]$. It has been hypothesized that motor thresholds depend on the excitability of the associated neural elements, which are excited by TMS and propagate the elicited action potential [19]. Therefore, RMT is considered as an excitability marker for the motor cortex [6]. UPDRSIII is a widely used measurement tool to assess the severity of motor dysfunction in parkinsonism patients because it provides a highly reliable and valid comprehensive assessment of motor symptoms [15]. In this study, the RMT of upper extremity MEPs exhibited a significant, negative relationship with the UPDRS-III score in patients with parkinsonism. These findings suggest that motor cortex excitability correlates with severity of motor dysfunction in patients with parkinsonism. The results of the present study were consistent with findings of previous studies 
on cortical hyper-excitability and reduced inhibition of the motor cortex in IPD [24-28]. Some studies have also reported that the motor threshold is associated with a lower RMT in IPD patients, as compared to the healthy population $[24,27,28]$. Therefore, the RMT of upper extremity MEPs could allow for quantitative measurement of motor dysfunction in patients with parkinsonism.

Upper and lower limb motor representations share similar mechanisms according to the study with paired pulse TMS paradigms [29], although intrinsic hand muscles with strong corticospinal projections have lower RMT than lower limb muscles with weaker corticospinal projections [30]. TMS-induced MEPs study in patients with IPD showed that there are similar abnormalities in intra-cortical facilitation and inhibition between the upper and lower extremity motor cortex [24]. A significant correlation between RMTs of MEPs in upper and lower extremities was consistent with previous studies [24,29]. Conversely, the RMT of MEPs in the lower extremity did not significantly correlate with severity of motor dysfunction, despite a significant relationship between RMT of MEPs in upper and lower extremities. The small number of participants in the study might be a possible reason of the tendency toward correlation between the RMT of MEPs in the lower extremity and severity of motor dysfunction, without statistical significance. However, functional activation across brain motor areas during lower extremity movements are similar but differ substantially from activation related to upper extremity movements [31]. Therefore, another reason might be that 2 kinds of movement differently recruit the central nervous system. Therefore, additional studies are needed to determine the precise relationship between the RMT of MEPs in the lower extremity and the severity of motor dysfunction.

The amplitude and latency of MEPs exhibited no significant relationship with functional assessments in patients with parkinsonism. In addition, there was no significant relationship between the latency of MEPs in the upper and lower extremities. The amplitude and latency of MEPs represent the corticospinal excitability of the M1 and the conduction time from the M1 to the target muscle, respectively [6]. The interpretation of MEP amplitude is complicated due to the influence of temporal dispersion of corticospinal conduction [6]. The latency of MEP might also be influenced by peripheral nerve conduction time, as well as central conduction time [6]. Therefore, the amplitude and latency in TMS-induced MEPs can be influenced by parameters such as height, functional status of peripheral nerves, and muscles [32]. This is one of limitations in the present study. Further studies to assess the exact relationship of the amplitude and latency of MEPs with functional assessments are needed. The RMT of MEPs is the most reliable parameter for assessing corticomotor excitability in TMS-induced MEPs, as the RMT of MEPs is relatively less influenced by other factors of corticomotor excitability $[6,33]$. This finding is consistent with previous studies.

This study had several limitations. By recruiting patients with the clinical syndrome of parkinsonism, we did not limit selection to a single disease entity $[8,9]$. The pathophysiological mechanisms of parkinsonism might differ according to disease, despite similar clinical features. Although the results of the present study were used to assess motor function in patients with parkinsonism, the power to draw conclusions based on disease entity is insufficient. In addition, most participants scored 3 or 4 on a modified Hoehn and Yahr scale. We did not enroll patients with mild or severe disease status. Therefore, the results of this study cannot be generalized to all patients with parkinsonism. Further investigations are required to supplement these limited findings.

In this study, RMT of upper extremity MEPs was significantly correlated with the UPDRS-III score in patients with parkinsonism. This result indicated that MEPs reflect the severity of motor dysfunction in patients with parkinsonism. This suggests that MEPs provide a quantitative, electrodiagnostic method to assess motor function in patients with parkinsonism.

\section{CONFLICT OF INTEREST}

No potential conflict of interest relevant to this article was reported.

\section{ACKNOWLEDGMENTS}

This study was supported by the Samsung Medical Center grant (No. CRO112051) and the National Research Foundation of Korea (NRF) grant funded by the Korea government (MSIP) (No. NRF-2014R1A2A1A01005128). 


\section{REFERENCES}

1. Bestmann S, Krakauer JW. The uses and interpretations of the motor-evoked potential for understanding behaviour. Exp Brain Res 2015;233:679-89.

2. Pennisi G, Ferri R, Cantone M, Lanza G, Pennisi M, Vinciguerra $\mathrm{L}$, et al. A review of transcranial magnetic stimulation in vascular dementia. Dement Geriatr Cogn Disord 2011;31:71-80.

3. List J, Kubke JC, Lindenberg R, Kulzow N, Kerti L, Witte V, et al. Relationship between excitability, plasticity and thickness of the motor cortex in older adults. Neuroimage 2013;83:809-16.

4. Di Lazzaro V, Oliviero A, Profice P, Ferrara L, Saturno E, Pilato F, et al. The diagnostic value of motor evoked potentials. Clin Neurophysiol 1999;110:1297-307.

5. Curra A, Modugno N, Inghilleri M, Manfredi M, Hallett M, Berardelli A. Transcranial magnetic stimulation techniques in clinical investigation. Neurology 2002;59:1851-9.

6. Groppa S, Oliviero A, Eisen A, Quartarone A, Cohen LG, Mall V, et al. A practical guide to diagnostic transcranial magnetic stimulation: report of an IFCN committee. Clin Neurophysiol 2012;123:858-82.

7. Kobayashi M, Pascual-Leone A. Transcranial magnetic stimulation in neurology. Lancet Neurol 2003;2:14556.

8. Wirdefeldt K, Adami HO, Cole P, Trichopoulos D, Mandel J. Epidemiology and etiology of Parkinson's disease: a review of the evidence. Eur J Epidemiol 2011;26 Suppl 1:S1-58.

9. Dickson DW. Parkinson's disease and parkinsonism: neuropathology. Cold Spring Harb Perspect Med 2012;2:a009258.

10. Kluger BM, Brown RP, Aerts S, Schenkman M. Determinants of objectively measured physical functional performance in early to mid-stage Parkinson disease. PM R 2014;6:992-8.

11. Salawu FK, Danburam A, Olokoba AB. Non-motor symptoms of Parkinson's disease: diagnosis and management. Niger J Med 2010;19:126-31.

12. Stebbins GT, Goetz CG. Factor structure of the Unified Parkinson's Disease Rating Scale: Motor Examination section. Mov Disord 1998;13:633-6.

13. Lee SY, Kim MS, Chang WH, Cho JW, Youn JY, Kim $\mathrm{YH}$. Effects of repetitive transcranial magnetic stimu- lation on freezing of gait in patients with Parkinsonism. Restor Neurol Neurosci 2014;32:743-53.

14. Kim YH, You SH, Ko MH, Park JW, Lee KH, Jang SH, et al. Repetitive transcranial magnetic stimulationinduced corticomotor excitability and associated motor skill acquisition in chronic stroke. Stroke 2006; 37:1471-6.

15. Movement Disorder Society Task Force on Rating Scales for Parkinson's Disease. The Unified Parkinson's Disease Rating Scale (UPDRS): status and recommendations. Mov Disord 2003;18:738-50.

16. Bryant MS, Rintala DH, Graham JE, Hou JG, Protas EJ. Determinants of use of a walking device in persons with Parkinson's disease. Arch Phys Med Rehabil 2014;95:1940-5.

17. Goetz CG, Poewe W, Rascol O, Sampaio C, Stebbins GT, Counsell C, et al. Movement Disorder Society Task Force report on the Hoehn and Yahr staging scale: status and recommendations. Mov Disord 2004;19:10208.

18. Nascimbeni A, Gaffuri A, Granella L, Colli M, Imazio P. Prognostic value of motor evoked potentials in stroke motor outcome. Eura Medicophys 2005;41:125-30.

19.Ziemann U, Reis J, Schwenkreis P, Rosanova M, Strafella A, Badawy R, et al. TMS and drugs revisited 2014. Clin Neurophysiol 2015;126:1847-68.

20. Appel-Cresswell S, de la Fuente-Fernandez R, Galley S, McKeown MJ. Imaging of compensatory mechanisms in Parkinson's disease. Curr Opin Neurol 2010;23:40712.

21. Sabatini U, Boulanouar K, Fabre N, Martin F, Carel C, Colonnese $\mathrm{C}$, et al. Cortical motor reorganization in akinetic patients with Parkinson's disease: a functional MRI study. Brain 2000;123(Pt 2):394-403.

22. Kojovic M, Kassavetis P, Bologna M, Parees I, RubioAgusti I, Beraredelli A, et al. Transcranial magnetic stimulation follow-up study in early Parkinson's disease: a decline in compensation with disease progression? Mov Disord 2015;30:1098-106.

23. Rossini PM, Barker AT, Berardelli A, Caramia MD, Caruso G, Cracco RQ, et al. Non-invasive electrical and magnetic stimulation of the brain, spinal cord and roots: basic principles and procedures for routine clinical application. Report of an IFCN committee. Electroencephalogr Clin Neurophysiol 1994;91:79-92.

24. Tremblay F, Tremblay LE. Cortico-motor excitability 
of the lower limb motor representation: a comparative study in Parkinson's disease and healthy controls. Clin Neurophysiol 2002;113:2006-12.

25. Bares M, Kanovsky P, Klajblova H, Rektor I. Intracortical inhibition and facilitation are impaired in patients with early Parkinson's disease: a paired TMS study. Eur J Neurol 2003;10:385-9.

26. Teo WP, Rodrigues JP, Mastaglia FL, Thickbroom GW. Modulation of corticomotor excitability after maximal or sustainable-rate repetitive finger movement is impaired in Parkinson's disease and is reversed by levodopa. Clin Neurophysiol 2014;125:562-8.

27. Suppa A, Iezzi E, Conte A, Belvisi D, Marsili L, Modugno N, et al. Dopamine influences primary motor cortex plasticity and dorsal premotor-to-motor connectivity in Parkinson's disease. Cereb Cortex 2010;20:2224-33.

28. MacKinnon CD, Gilley EA, Weis-McNulty A, Simuni T. Pathways mediating abnormal intracortical inhibition in Parkinson's disease. Ann Neurol 2005;58:516-24.

29. Chen R, Tam A, Butefisch C, Corwell B, Ziemann U,
Rothwell JC, et al. Intracortical inhibition and facilitation in different representations of the human motor cortex. J Neurophysiol 1998;80:2870-81.

30. Brouwer B, Ashby P. Corticospinal projections to upper and lower limb spinal motoneurons in man. Electroencephalogr Clin Neurophysiol 1990;76:509-19.

31. Luft AR, Smith GV, Forrester L, Whitall J, Macko RF, Hauser TK, et al. Comparing brain activation associated with isolated upper and lower limb movement across corresponding joints. Hum Brain Mapp 2002;17:131-40.

32. Matamala JM, Nunez C, Lera L, Verdugo RJ, Sanchez $\mathrm{H}$, Albala C, et al. Motor evoked potentials by transcranial magnetic stimulation in healthy elderly people. Somatosens Mot Res 2013;30:201-5.

33. Lewis GN, Signal N, Taylor D. Reliability of lower limb motor evoked potentials in stroke and healthy populations: how many responses are needed? Clin Neurophysiol 2014;125:748-54. 